\title{
LV-DB-3
}

\section{Hepatocellular carcinoma with portal vein invasion: Non-surgical treatment}

\author{
${\underline{B o ~ H y u n ~} \mathrm{KIM}^{*}}^{*}$ \\ Division of Gastroenterology, Department of Internal Medicine, Center for Liver and Pancreatobiliary Cancer, \\ National Cancer Center, Goyang, Korea
}

Lecture: A substantial proportion of patients with hepatocellular carcinoma (HCC) present with portal vein invasion (PVI). The extent of portal vein tumor thrombosis (PVTT) can be classified into 4 categories: Vp1, existence of PVTT distal to the second-order branches of portal vein; Vp2, existence of PVTT in the second-order branches of portal vein; VP3, PVTT in the first-order branches of portal vein; and VP4, existence of PVTT in main portal trunk or portal branches contralateral to primary involved lobe. The degree of PVTT has shown the close association with prognosis of HCC across all treatment options.

Clinical practice guidelines recommend systemic therapy for advanced HCC with PVI based on the highest level of evidence. Sorafenib has firstly shown to improve overall survival of patients with advanced HCC in 2007 [1]. Among 299 sorafenib-treated patients, 108 (36\%) had macrovascular invasion. Subgroup analysis consistently demonstrated significantly longer overall survival (8.1 vs. 4.9 months; hazard ratio [HR], 0.68; 95\% confidence interval [CI], 0.49-0.93) and time to progression (4.1 vs. 2.7 months; HR, 0.57 ; 95\% CI 0.39-0.84) in sorafenib-treated patients with macrovascular invasion [2]. Lenvatinib, another 1st-line systemic agent, has proven non-inferiority to sorafenib in advanced HCC [3]. The REFLECT study included patients with macroscopic portal vein invasion (199/954, 21\%); however, it excluded patients with main portal vein invasion. Recently approved systemic agents, atezolizumab plus bevacizumab was superior to sorafenib in overall survival and progression-free survival [4]. Of 336 atezolizumab-bevacizumab treated patients, $38 \%$ had macrovascular invasion. Atezolizumab with bevacizumab also showed significant efficacy in patients with microvascular invasion (HR 0.58 [95\% CI, 0.38-0.89] for overall survival; HR 0.53 [95\% CI, 0.37-0.76] for progression-free survival).

Transarterial therapy and/or radiotherapy can also be an option for HCC with PVI. A single-center randomized controlled study has demonstrated that transarterial chemoembolization with external beam radiotherapy significantly improved progression-free survival and overall survival compared with sorafenib in patients with macroscopic vascular invasion [5]. Earlier studies reported that transarterial chemoembolization improved overall survival of HCC patients with PVTT compared to supportive care [6,7] Recent meta-analysis indicated that radioembolization was safer and more effective than sorafenib in HCC with PVI [8].

\section{References}

1. Llovet JM, Ricci S, Mazzaferro V, Hilgard P, Gane E, Blanc JF, et al. Sorafenib in advanced hepatocellular carcinoma. N Engl J Med 2008;359:378-390.

2. Bruix J, Raoul JL, Sherman M, Mazzaferro V, Bolondi L, Craxi A, et al. Efficacy and safety of sorafenib in patients with advanced hepatocellular carcinoma: subanalyses of a phase III trial. J Hepatol 2012;57:821-829.

3. Kudo M, Finn RS, Qin S, Han KH, Ikeda K, Piscaglia F, et al. Lenvatinib versus sorafenib in first-line treatment of patients with unresectable hepatocellular carcinoma: a randomised phase 3 non-inferiority trial. Lancet 2018;391:1163-1173.

4. Finn RS, Cheng AL. Atezolizumab and Bevacizumab in Hepatocellular Carcinoma. Reply. N Engl J Med 2020;383:695.

5. Yoon SM, Ryoo BY, Lee SJ, Kim JH, Shin JH, An JH, et al. Efficacy and Safety of Transarterial Chemoembolization Plus External Beam Radiotherapy vs Sorafenib in Hepatocellular Carcinoma With Macroscopic Vascular Invasion: A Randomized Clinical Trial. JAMA Oncol 2018;4:661-669.

6. Luo J, Guo RP, Lai EC, Zhang YJ, Lau WY, Chen MS, et al. Transarterial chemoembolization for unresectable hepatocellular carci- 
noma with portal vein tumor thrombosis: a prospective comparative study. Ann Surg Oncol 2011;18:413-420.

7. Chung GE, Lee JH, Kim HY, Hwang SY, Kim JS, Chung JW, et al. Transarterial chemoembolization can be safely performed in patients with hepatocellular carcinoma invading the main portal vein and may improve the overall survival. Radiology 2011;258:627634.

8. Kim PH, Choi SH, Kim JH, Park SH. Comparison of Radioembolization and Sorafenib for the Treatment of Hepatocellular Carcinoma with Portal Vein Tumor Thrombosis: A Systematic Review and Meta-Analysis of Safety and Efficacy. Korean J Radiol 2019;20:385-398. 